\title{
Medical Marijuana Efficacy: A Survey
}

\author{
Ngugi M. Kinyungu ${ }^{*}$, Josiah Gikungi ${ }^{2}$, Ariel Davson ${ }^{3}$ \\ ${ }^{1}$ ITAV Pain Management, Donald and Barbara Zucker School of Medicine at Hofstra/Northwell, New York, USA \\ ${ }^{2}$ Elson S. Floyd College of Medicine, Spokane, Washington DC, USA \\ ${ }^{3}$ St. Georges University, Grenada \\ Email: ^mukorak@yahoo.com
}

How to cite this paper: Kinyungu, N.M., Gikungi, J. and Davson, A. (2022) Medical Marijuana Efficacy: A Survey. Open Journal of Anesthesiology, 12, 91-98. https://doi.org/10.4236/ojanes.2022.123008

Received: February 6, 2022

Accepted: March 5, 2022

Published: March 8, 2022

Copyright $\odot 2022$ by author(s) and Scientific Research Publishing Inc. This work is licensed under the Creative Commons Attribution International License (CC BY 4.0).

http://creativecommons.org/licenses/by/4.0/ (c) (i) Open Access

\begin{abstract}
Background: Medical marijuana use has seen a rise and acceptance in the last several years and while its efficacy has been spoken and discussed in lore, data on efficacy still remains scarce. Aim: We conducted a survey on chronic pain patients certified for medical marijuana to assess pain relief and efficacy. Findings: Our survey found that the pain score with the use of medical marijuana was halved in our 20 respondents and sleep was improved. Overall function improved. Conclusion: The survey shows that medical marijuana is efficacious in chronic pain patients and further studies need to be done and access to the medication needs to be improved so as to benefit more patients.
\end{abstract}

\section{Keywords}

Medical Marijuana, Cannabis, Chronic Pain

\section{Introduction}

As the opioid epidemic continues, different approaches to pain management are required so as to reduce the amounts of opioid prescribed to patients suffering from chronic pain. One therapeutic option that has been considered is cannabis and multiple states today have instituted a medical marijuana program for different conditions, one of those being chronic pain. Marijuana exerts its effects through the endocannabinoid system which has 2 types of receptors: type 1 (CB1) and 2 (CB2) cannabinoid receptors. CB1 mediates the neuropsychiatric effects of marijuana as the receptor is expressed in the central nervous system. CB2 is expressed in peripheral blood mononuclear cells [1]. Multiple studies have demonstrated an association between marijuana legalization with reductions in rates of opioid overdose and opioid-related hospitalizations among other outcomes [2]. 
In July 2014, the Compassionate Care Act was enacted in the state of New York allowing the establishment of a medical marijiuana program to meet the needs of residents of the state. To be able to participate, patients had to get certification from a certified practitioner and subsequently get the marjuana from licensed dispensaries spread throughout the state. Chronic pain was not initially one of the conditions recognized under the compassionate care act but after an outcry by the public, chronic pain was added as a qualifying condition for medical marijuana [3].

Chronic pain was thus included in the list of the ten other qualifying conditions. The ten other qualifying conditions included Cancer, HIV infection, ALS, Parkinson's disease, multiple sclerosis, damage to the nervous tissue of the spinal cord with objective neurological indication of intractable epilepsy, inflammatory bowel disease, neuropathies and Huntington's disease. With the legalization of medical marijuana in most states and recreational marijuana in some states, more studies are needed to assess the efficacy of marijuana as a means of addressing chronic pain, cachexia/wasting syndrome, glaucoma among others [1]. As part of efforts to improve healthcare and improve the data we have on the use of medical marijuana, we conducted a study of the efficacy of medical marijuana for chronic pain in an outpatient chronic pain clinic setting.

\section{Materials and Methods}

\section{Sample Selection.}

The study was carried out in an outpatient pain clinic setting in New York from October 2018 to May 2019.

Selection criteria: All patients who were on medical marijuana and were returning for their follow up visits between the period October 2018 and May 2019 were offered the survey. Patients under the age of 18 were excluded from this survey. Survey answers were collected in the clinic, via secure email and phone calls. We had a total of 21 participants who responded to the request.

The study qualified for exempt status. As defined in the federal regulations: minimal risk standard was applied: 'the probability and magnitude of harm or discomfort anticipated in the research are not greater in and of themselves than those ordinarily encountered in daily life or during the performance of routine physical or psychological examinations or tests. Per electronic code of Federal Regulations in effect August, 2018, Research activities involving human subjects

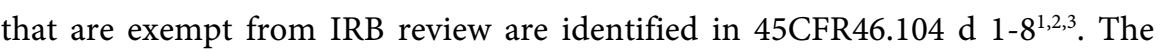
Office of Human Research Protections (U.S. Department of Health and Human Services) does not specify who at an institution may determine that research is exempt but recommends that, because of the potential for conflict of interest, investigators not be given the authority to make an independent determination

\footnotetext{
${ }^{1}$ https://www.hhs.gov/ohrp/regulations-and-policy/regulations/45-cfr-46/index.html ${ }^{2}$ https://www.ecfr.gov/cgi-bin/text-idx? $\mathrm{m}=08 \& \mathrm{~d}=10 \& \mathrm{xy}=2018 \& \mathrm{~cd}=20200820 \&$ submit $=\mathrm{GO} \& S I D=8 \mathrm{~cd}$ c4f9cdec93e62c16a7d57d8363b48\&node=pt45.1.46\&pd=20180806\#se45.1.46_1104

${ }^{3}$ https://www.accessdata.fda.gov/scripts/cdrh/cfdocs/cfcfr/CFRSearch.cfm?fr=56.102
} 
that human subject research is exempt ${ }^{4}$. Per this recommendation by OHRP, this survey was reviewed by an independent reviewer who had sufficient information to make a decision and deemed the study exempt as identified in 45CFR46.104 d2. Belmont principle ${ }^{5}$ of Respect for Persons generally requires that subjects be given the opportunity to choose whether or not to participate in research. In line with this principle, Voluntary informed consent was obtained from participants for this exempt survey. Also per recommendation by OPHS (Office of Public Health and Science) the participants were provided with the following minimum consent information: 1) The identity and affiliation of the researcher. 2) A clear description of the study procedures and how data will be used in the future. 3) A statement that participation in the research is voluntary. 4) Contact information for questions about the research.

\section{Survey Design and administration:}

All patients were asked to answer the following questions:

1) What is your overall pain score before using the medication and afterwards on a scale of $1-10$ ?

2) How much relief would you say you have had as a percentage, e.g. $50 \%$ overall relief?

3) Is your sleep improved and if so by how much as a percentage, e.g. $50 \%$ better?

4) Has your overall function improved and if so by how much as a percentage, e.g. $40 \%$ better (function included aspects like walking, cooking etc. activities of daily living)?

5) Any improvement in other symptoms not mentioned above?

6) Any comments you would like to make on use of medical marijuana?

\section{Results}

The study included 21 respondents: one survey was blank when returned. There were 11 females and 10 males. The patients aged from 31 to 71 with the average being 48.59. All the patients have chronic pain.

\section{Survey question number 1 :}

Survey question number 1 asked the average pain score before and after use of medical marijuana. (See the survey questions in the appendix section.)

Most participants reported significant improvement in pain relief after the use of marijuana as shown in Figure 1 and Table 1).

Average pain score before was 8.4 and average pain score after was 4.275.

\section{Survey question number 2 :}

Survey question number 2 queried the percentage relief patients experienced.

The participants reported an average 55\% pain relief after administration of marijuana as shown in Figure 2.

\section{Survey question number 3:}

Survey question number 3 queried the patients on improvement in sleep as a

${ }^{4}$ https://www.hhs.gov/ohrp/regulations-and-policy/guidance/faq/exempt-research-determination/in dex.html\#4109

${ }^{5}$ https://cphs.berkeley.edu/exempt.pdf 
percentage.

19 out of the 21 patients reported improvement in sleep ranging from 15\% $100 \%$.

\section{Overall Pain for all patients}

$\square$ Before $\square$ After

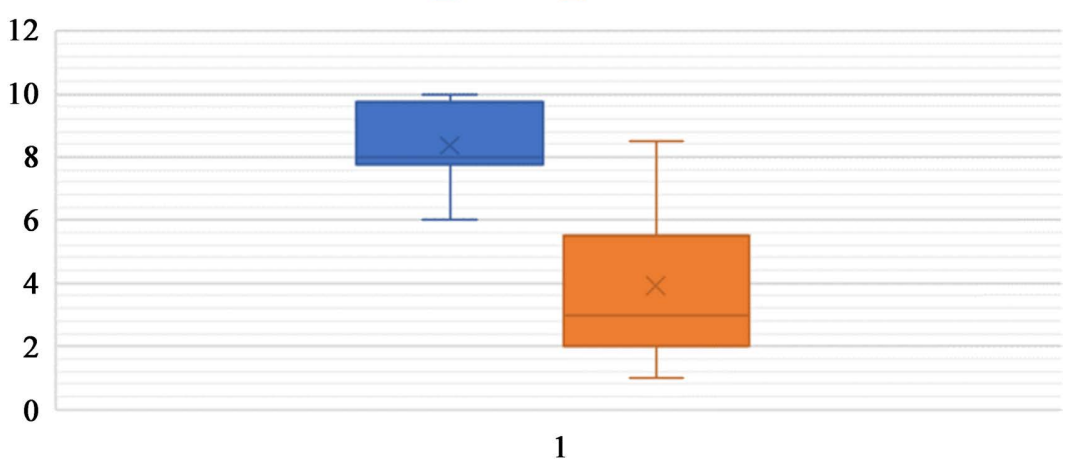

Figure 1. Average pain score before and after use of medical marijuana.

Table 1. Average pain score before and after use of medical marijuana.

\begin{tabular}{|c|c|c|c|}
\hline participant & pain before & pain after & age \\
\hline 1 & 8 & 2 & 61 \\
\hline 2 & 7 & 2 & 56 \\
\hline 3 & 10 & 2 & 53 \\
\hline 4 & 8 & 5 & 59 \\
\hline 5 & 9 & 5 & 31 \\
\hline 6 & 10 & 8.5 & 28 \\
\hline 7 & 8 & 3 & 71 \\
\hline 8 & 8 & 3 & 66 \\
\hline 9 & 8 & 8 & 57 \\
\hline 10 & 9 & 6 & 64 \\
\hline 11 & 9 & 5 & 49 \\
\hline 12 & 8 & 4 & 51 \\
\hline 13 & 10 & 2 & 37 \\
\hline 14 & 10 & 5 & 35 \\
\hline 15 & 8 & 3 & 57 \\
\hline \multicolumn{4}{|l|}{16} \\
\hline 17 & 10 & 8 & 49 \\
\hline 18 & 6 & 2 & 37 \\
\hline 19 & 8 & 3 & 33 \\
\hline 20 & 7 & 3 & 59 \\
\hline 21 & 7 & 6 & 31 \\
\hline
\end{tabular}




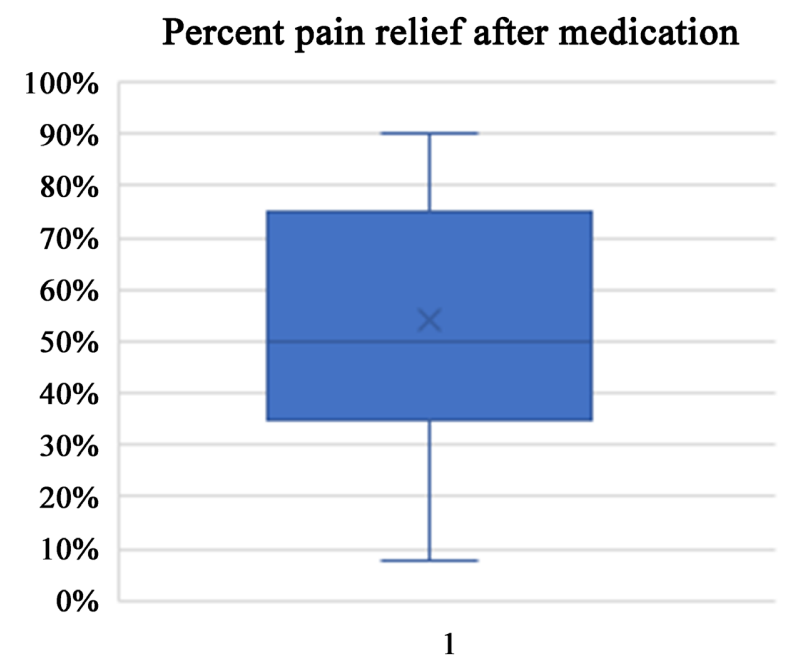

Figure 2. Percentage pain relief after use of medical marijuana.

\section{Survey question number 4 :}

Survey question number 4 queried the patients on improvement in daily function as a percentage

18 out of the 21 patients observed improvement in daily functioning ranging from $10 \%$ to $85 \%$. Survey question number 5 :

Survey question number 5 asked the patients to report any other improvements on the medical marijuana.

16 participants reported other improvements that were not assessed in the survey. They noticed decreased levels of anxiety, increased appetite, decreased migraines, reduced swelling, improvement in mood as well as an increased quality of life. 5 participants did not report any other improvements.

\section{Survey question number 6}

Survey question number 6 asked the patient to make any other comments on the use of medical marijuana.

We had ten responses to this open ended question. See the responses in the addendum section.

\section{Discussion}

Chronic pain is one of the most common reasons for prescription of medical marijuana. Even though clinical data supports the use of marijuana for pain, there is still not much known about the efficacy, dose, route of administration, or the adverse effects [1].

Medical marijuana offers numerous benefits such as better management of pain as demonstrated by the participants of this study. The responses in this study showed a 50\% reduction in pain levels after use of medical marijuana. Other benefits that we observed include decreased levels of anxiety, increased appetite, decreased migraines, reduced swelling, improvement in mood as well as an increased quality of life as reported on the surveys. The improvement in sleep evidenced in this study is significant as poor sleep worsens overall pain 
scores and also interacts negatively on aspects of health including mental health [4]. Marijuana is also relatively non-addicting, and few withdrawal effects have been reported with perhaps one of the most significant benefits of medical marijuana being the reduction in opioid overdose mortality rates [5].

There are limitations to this study, the first being that the patients who responded were patients already on medical marijuana and returning for follow up visits and hence they already had benefit from the medical marijuana. This is also a survey as opposed to a case controlled study with an interventional study group. Marijuana remains a federal class 1 drug which limits such studies. There is therefore lack of longitudinal studies evaluating the effects of chronic marijuana use.

\section{Conclusion}

This study offers a brief but enlightening view on the efficacy of medical marijuana among patients. The study showed evidence of efficacy with use of medical marijuana with a $50 \%$ reduction in pain scores, improved sleep and improvement in overall daily functioning. This study focused on efficacy but did not focus on the side effects and adverse reactions after using medical marijuana. The use of medical marijuana continues to be accepted both by patients and the medical community as evidenced by the number of states in the USA that have systemized the usage of medical marijiana for their residents. Looking ahead, more studies are necessary to fully delineate the efficacy of medical marijiana, side effects and dosing.

\section{Conflicts of Interest}

The authors declare no conflicts of interest regarding the publication of this paper.

\section{References}

[1] Ebbert, J.O., Scharf, E.L. and Hurt, R.T. (2018) Medical Cannabis. Mayo Clinic Proceedings, 93, 1842-1847. https://doi.org/10.1016/j.mayocp.2018.09.005

[2] Wendelboe, A.M., Mathew, R., Chongsuwat, T., Rainwater, E., Wendelboe, M.A., Wickersham, E. and Chou, A.F. (2019) Is There Less Opioid Abuse in States Where Marijuana Has Been Decriminalized, Either for Medicinal or Recreational Use? A Clin-IQ. Journal of Patient-Centered Research and Reviews, 6, 267-273. https://doi.org/10.17294/2330-0698.1704

[3] (2016) NYSDOH Announces Chronic Pain to Be Added as Qualifying Condition for Medical Marijuana, Albany, NY, Dec. 1. https://www.health.ny.gov/press/releases/2016/2016-12-01 chronic pain condition added.htm

[4] Zambelli, Z., Halstead, E., Fidalgo, A.R. and Dimitriou, D. (2021) Good Sleep Quality Improves the Relationship between Pain and Depression among Individuals with Chronic Pain. Frontiers in Psychology, 12, Article ID: 668930. https://doi.org/10.3389/fpsyg.2021.668930

[5] Webb, C.W. and Webb, S.M. (2014) Therapeutic Benefits of Cannabis: A Patient Survey. Hawai $i$ Journal of Medicine \& Public Health, 73, 109. 


\section{Addendum}

\section{Survey Questions:}

survey question number 1: What is your overall pain score before using the medication and afterwards on a scale of 1 - 10.

survey question number 2: How much relief would you say you have had as a percentage, e.g. $50 \%$ overall relief

survey question number 3: Is your sleep improved and if so by how much as a percentage, e.g. $50 \%$ better

survey question number 4: Has your overall function improved and if so by how much as a percentage, e.g. 40\% better ( function included things like walking, cooking etc. activities of daily living)

survey question number 5: Describe any Improvement in other symptoms not mentioned above

survey question number 6: Any comments you would like to make on use of medical marijuana:

Responses to survey question number 6 (Any comments you would like to make on use of medical marijuana)

Response 1: (for medical marijuana) to be cheaper and more affordable and for insurance to cover it.

Response 2: I hope New York adds additional ways to customize based on individual needs. Right now only 3 types of formulas available.

$3^{\text {rd: }}$ The government needs to realize and accept the fact that herbs naturally grown from the earth are medicinal and we don't need the chemically synthesized drugs to help reduce pain.

$4^{\text {th. }}$ It has helped me live a semi normal life by being able to do things that I haven't been able to do due to constant chronic pain. It's the only medicine that has truly worked and helped me.

$5^{\text {th: }}$ It would be a great help if New York State would approve the use of flower and edible forms of medication as well as allowing patients to grow their own plants.

$6^{\text {th }}$ : After being on prescription opioids for over 5 years due to multiple surgeries, medical marijuana clearly aided me in eliminating opioids from my daily treatment. I am no longer taking any opioids for pain management, and while there are side effects when using medical marijuana just like prescription drugs, I feel much better both physically and mentally.

$7^{\text {th }}$ : Utilizing medical marijuana has allowed me to regain so much functioning that I did not have prior due to chronic pain and how exhausting that is on my body. The 1:20 CBD I use during the day provides pain relief without side effects so I can continue to work without being in pain. The 20:1 THC vape has been excellent help for me when I cannot fall asleep/stay asleep, but what I appreciate most is the 20:1 THC pills that I take that allow me to sleep through the night without waking up from pain and then being exhausted and even more achy the following day. 
$8^{\text {th }}$ Yes, all the benefits of marijuana need to be spread, no one needs to be taking opioids and narcotic medications and become addicted to them when this natural medicine is available.

$9^{\text {th }}$ : Very sensitive to THC with severe symptoms if not extremely cautious. Must take in combination with OTC CBD oil to minimize unpleasant THC effects. CBD by itself (Rx or OTC) no effect.

$10^{\text {th: }}$ : Unfortunately I am unable to use the products as frequently as I wish I could, especially during the day time knowing that I do have to operate a vehicle. I do know $100 \%$ that using the products helps me when I am in pain, especially at night time when pain is usually at its peak. 